\title{
Online Metallic Surface Defect Detection Using Deep LearningOnline Metallic Surface Defect Detection Using Deep Learning
}

Feyza ÇEREZCi ( $\nabla$ feyzacerezci@sakarya.edu.tr )

Sakarya Universitesi https://orcid.org/0000-0002-1596-1109

\section{Serap KAZAN}

Sakarya Universitesi

Muhammed Ali Oz

Yildiz Teknik Universitesi

Cemil Oz

Sakarya Universitesi

Tugrul Tasci

Sakarya Universitesi

Selman Hızal

Sakarya Universitesi

Çağlayan Altay

Sakarya Universitesi

\section{Original Article}

Keywords: deep learning, defect detection, CNN, photometric stereo, machine learning

Posted Date: July 16th, 2020

DOI: https://doi.org/10.21203/rs.3.rs-41274/v1

License: (9) This work is licensed under a Creative Commons Attribution 4.0 International License.

Read Full License 


\title{
Online Metallic Surface Defect Detection Using Deep Learning
}

\author{
Feyza Cerezci - Serap Kazan · Muhammed Ali Oz • Cemil Oz • Tuğrul Tasci • Selman Hızal • \\ Çağlayan Altay
}

Received: date / Accepted: date

\begin{abstract}
Across a range of manufacturing contexts, automated quality control has been gaining significant attention because it offers competitive advantages such as cost reduction, high accuracy in defect detection, and system stability over time. Although computer vision has historically been the most commonly applied method in this context, novel approaches such as deep learning have recently become more frequent and are used in cases where traditional methods cannot be applied. Because of the surface texture and curvature of many metallic parts, detection of defects such as
\end{abstract}

Feyza Cerezci*

Computer Engineering, Sakarya University, Sakarya, TURKEY

Corresponding Author E-mail: feyzacerezci@sakarya.edu.tr

Serap Kazan

Computer Engineering, Sakarya University, Sakarya, TURKEY

E-mail: scakar@sakarya.edu.tr

Muhammed Ali Oz

Control and Automation Engineering, Yıldiz Technical University, Istanbul, TURKEY

E-mail: maoz@yildiz.edu.tr

Cemil Oz

Computer Engineering, Sakarya University, Sakarya, TURKEY

E-mail: coz@sakarya.edu.tr

Tuğrul Tasci

Information System Engineering, Sakarya University,

Sakarya, TURKEY

E-mail: ttasci@sakarya.edu.tr

Selman Hizal

Computer Engineering, Sakarya University, Sakarya, TURKEY

E-mail: shizal@sakarya.edu.tr

Çă̆layan Altay

Cengiz Machine Company

E-mail: caltay@cengizmakina.com.tr scratches, cracks, and dents can be challenging for traditional computer vision methods. In this study, an image acquisition system supported by a special lighting device that provides processable images from an extremely reflective cylindrical metallic surface has been developed. Multiple images obtained from a single lateral line of the surface, which is rotated at a specified speed, are combined using photometric stereo and given as input to a convolutional neural network that is employed to classify defective and non-defective samples. The results obtained from this method are close to $98.5 \%$ accurate.

Keywords deep learning · defect detection · CNN . photometric stereo $\cdot$ machine learning

\section{Introduction}

In recent years, the need for automatic defect detection systems that operate during the production phase of manufacturing has been increasing. Quality control procedures are used to identify the defects in products and to determine the locations of these defective areas. Defect detection is both important and necessary in manufacturing because producing flawed products can result in substantial economic losses. Although human vision is still the traditional method used for this purpose during production, computer vision techniques are more effective than human detection systems [1].

Quality control systems based on computer vision are applied in situations where it is difficult to quickly and accurately distinguish errors with the human eye and where the process cost is unreasonable in the long term. However, the most important attribute for such a system is an image acquisition mechanism suitable for error detection via image processing methods. The 
performance of the image acquisition process depends on the coherent operation of many factors, including the ability to detect errors in object properties such as shape, geometry, and raw material. Both the flexible capability of the image acquisition device and the object lighting are of primary importance.

In terms of production performance, it is important to employ a real-time system for the detection of metal defects early in the manufacturing process. Using computer vision to detect defects in images of metal parts contributes to overall performance in a positive way by providing faster and more successful outcomes. Although the quantity of data is large, such a system can efficiently and effectively analyze errors that are too small to be seen without computer-aided systems [2].

Computerized image processing techniques are frequently used for defect detection in industrial process methods. There are numerous studies that describe feature extraction and classification to detect surface defects on image data [1-11]. Generally, inspection system are divided into three main stages: image acquisition, feature extraction, and classification. Woodham introduced photometric stereo for 3D analysis of surfaces with Lambertian reflection [12]. This method is capable of deriving the normal vectors of a surface from multiple images of that surface under different lighting conditions. Currently, photometric stereo is used to detect defects in many different products, including steel strips [13], and pharmaceutical tablets [14]. Deep learning was introduced into defect detection with photometric stereo to increase performance by improving the accuracy of the model and by decreasing the run time of the photometric stereo algorithm. The inverse reflectance model [15], the Gaussian process Model [16] and the YOLO model [17] were trained with datasets obtained from the output of the photometric stereo method.

Ghorai et al. extracted the features from the metallic part by using different wavelet transformations such as Haar, Daubechies 2 (DB2) and Daubechies 4 (DB4) and then classified them with support vector machines (SVMs) [18]. Zheng, Kong, and Nahavandi achieved successful results in defect detection by applying morphological processes and genetic algorithms [19]. Malekian et al. used the geometrical features of an image for feature extraction and artificial neural networks as classifiers [20].

In these classical approaches, feature extraction is performed using hand-selected features such as grayscale, geometric shape, texture, local binary pattern, wavelet transform, or their combinations and is generally followed by a classifier such as an artificial neural network, SVM, or similar algorithm [21].
With the improvement of artificial intelligence technology, classical approaches have achieved moderate results. However, these defect classification approaches primarily depend on the programmer or user to specify feature characteristics and lack the ability to employ self-learning and self-improvement methods. In order to overcome the above shortcomings, in this paper defect classification of metallic parts was performed using a convolutional neural network (CNN), which can directly learn better representative features from the labeled images of surface defects with supervised learning. A max-pooling CNN approach was tested for supervised steel defect classification and compared to the results of SVM classifiers [22]. Ye et al. obtained images from control devices that were trained with CNN [23].

In this study, the photometric stereo technique was used to prevent non-depth image content such as fingerprints and smudges from being processed. Then images were taken by illuminating the metal parts in 4 different directions. Afterwards, these images were pre-processed with photometric stereo, joined into a single image, and given to the input layer of $\mathrm{CNN}$.

The rest of the paper is organized as follows: Section 2 describes the basic methodology, the experiments are discussed in Section 3, and conclusions are drawn in Section 4.

\section{Methodology}

\subsection{Data Acquisition and Pre-Processing}

The objects to be imaged for error detection can be curved, as well as having a structure that cannot be viewed from every angle or that includes very small surfaces. In addition, objects consisting of different raw materials such as metals, glass, textiles, and plastics can have different sensitivities to ambient lighting; metal objects in particular may have very high reflectivity. In order to obtain an image from the objects being detected from the inner-outer or lateral surfaces, it is necessary to create flexible, non-vibratory, well-connected equipment that can be moved and angled and that can also support objects of different sizes. It is also important that the field of view be adjustable, such as by raising, lowering, and rotation. In addition, it is of great importance for the performance of the computer vision-based quality control systems to have cameras and lenses that are capable of obtaining high-resolution images of extremely small objects at very close range.

Also, ambient lighting is critical to the performance of the image acquisition process during image processing-based error detection. The process should 
incorporate a dominant lighting component to eliminate any unwanted light from objects or from the environment. It must also illuminate the target regions in the image acquisition area in a uniformly distributed manner.

In most cases, computer-aided quality control systems that are expected to be used for industrial purposes aim to detect defects in a large number of massproduced parts. This requires that the process of acquiring images occur in real time or in a pre-determined amount of time. Therefore, the process of taking images must be structured in such a way that a certain number of pieces are processed and classified within specified time intervals. In this context, it is a fundamental requirement that the number of parts that will be detected in a cycle pass through the system quickly. In order to meet such a requirement, a system capable of synchronously controlling the encoder, motor, lighting, and image processing software should be designed.

A mechanism consisting of many joints, which can be rotated and moved in different dimensions, is designed to accurately scan rounded products. The joints of the mechanism are used to optimize the position of the product so that it offers ideal visibility to the camera and is also efficiently illuminated by the light. Our image acquisition system and the controlled area of metal are shown in Figure 1. The metallic part under inspection is a component of a diesel-injection system used for transferring high-pressure fuel to the injector.

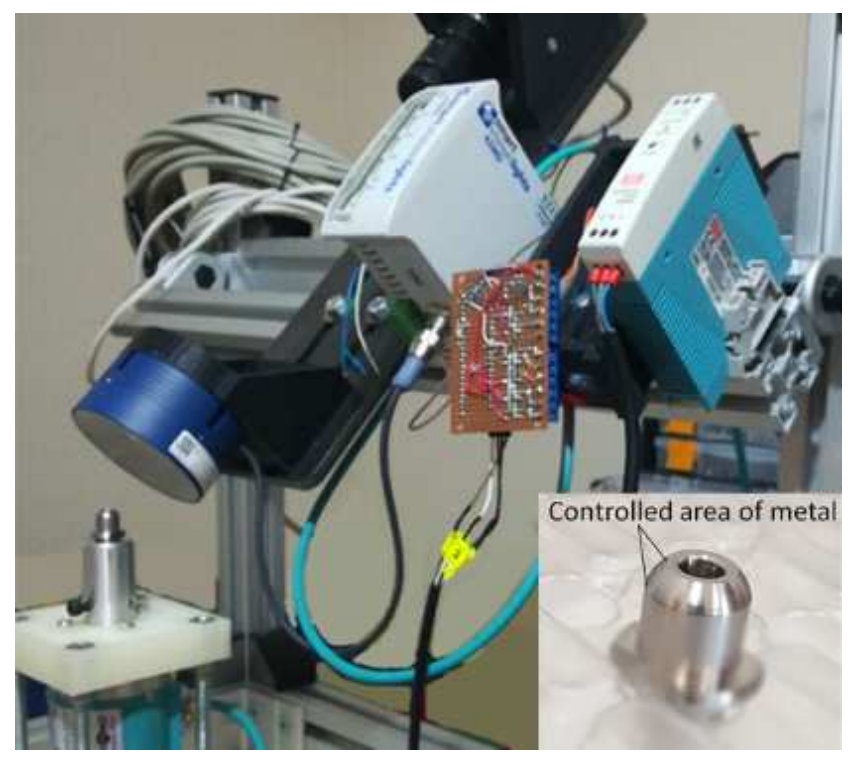

Fig. 1 Image acquisition system and controlled area of metal

The device was initially designed and simulated in three dimensions in a digital environment. Then, some parts were created with the help of three-dimensional printers, while other parts were produced by industrial enterprises.

In this study, an illumination system that can be controlled by an electronic control board is used. This system minimizes difficulties with light reflecting from metallic surfaces from which images will be acquired and provides strong, evenly distributed ambient light.

The system shown in Figure 1 includes LEDs and can control parameters such as their blink times and brightness. An industrial line scan camera is used for high accuracy image acquisition and error detection, along with an extension tube and lenses with a clear view of the small metallic surface from close up.The block diagram for the equipment used in the study, such as the camera, lighting, encoder and motor, is shown in Figure 2 .

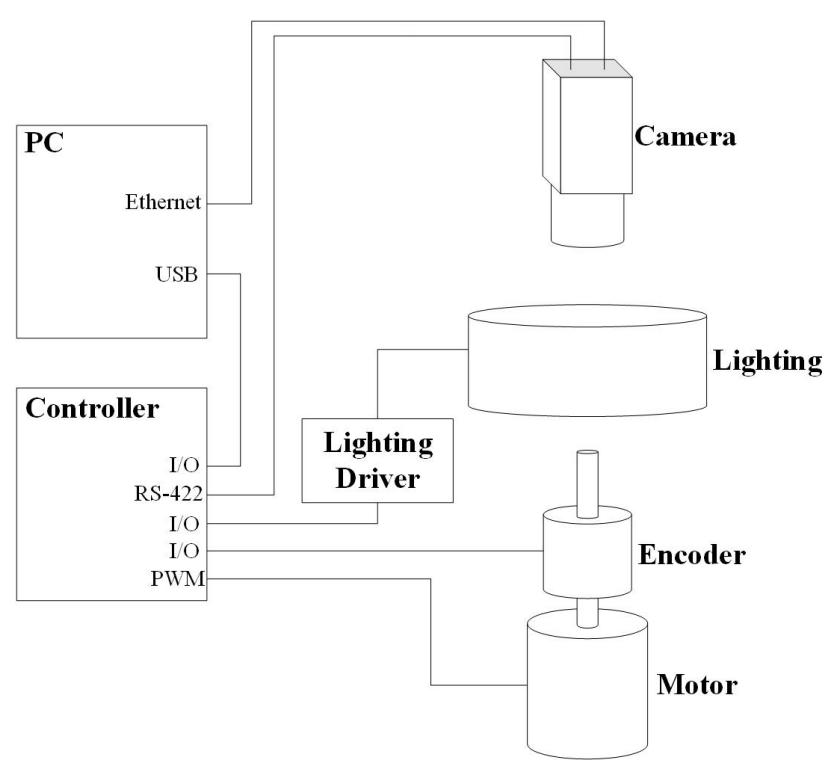

Fig. 2 Block diagram of the system

To support the defect detection algorithm, a hollow ring lighting apparatus that can create a radial lighting area is used. It can be controlled separately, has high power LED regions, and allows high trigger rates. A small strip belonging to the curved upper surface of the metallic part is imaged with the line scan camera positioned at a quasi-straight angle to this surface to minimize the reflection of unwanted light.

In order to display the surface properly in thin strips, the object is rotated with the help of a motor, and its travel while rotating is measured very precisely with the help of an encoder. By means of the controller, the system works in a coherent and accurate way. Following the start command from a PC, the controller drives the motor at constant speed with the pulse width modulation signal. It receives illuminated images from 
different directions from the same acceptable strip of the surface via signals from the encoder.

This process is carried out four times per surface strip so that the different lighting zones are lit in four ways. During this time, the accumulated images in the camera are transferred to the $\mathrm{PC}$ at the end of the image acquisition process and are passed through the defect detection algorithm.

For testing, the obtained metal parts were checked manually and then separated into defective and nondefective groups. Images were then taken with our system and kept in two separate folders. The final image for each part contains four sub-images of the same surface under different lighting. These sub-images are used as input to the photometric stereo methods explained in Chapter 2.2. Sample images are shown in Figure 3. Output images from the photometric stereo algorithm are provided as input to $\mathrm{CNN}$.
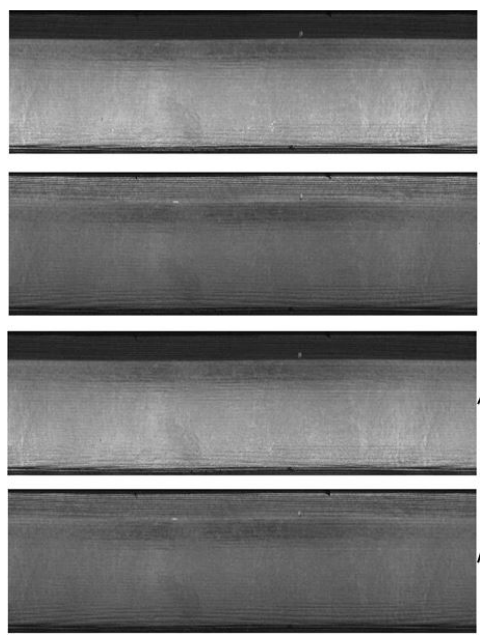

Fig. 3 Four images produced after pre-process phase

This research used CNN as our method. First, experts sorted the metal parts manually as described above. This step generated primary data by defining whether the metal parts were defective or not. We labeled this data as image "Ok" or "Nok." Then we resized and cropped the labeled parts images to $250 \times 250$ element pictures that showed only the full metal surface. The next step was the classification process using the CNN architecture. The training process is the stage in which CNN is trained to high accuracy when performing classification. During the training process, weights were updated until the error rate reached the desired level. The testing process, which uses bias and weight from the results of the training stage, is a classification process [24]. To recap, the training phase determined how to label images as "defective" and "non-defective," and the testing process classified images as "defective" or "non-defective" based on the training. Figure S1 of the Supplementary file shows the classification process.

\subsubsection{Photometric Stereo}

Photometric stereo (PS) is used to estimate the local surface normals by working with several images of the same surface taken from the same observation angle but under different illumination directions (as shown in Figure S2 of the Supplementary file). Ideal light sources are positioned so that light is received from different directions. Thus, in each case there is a well-positioned light source that assist in measuring the direction of the surface normal. The number of light sources can be as many as needed to determine accuracy, but a minimum of three light sources is needed.

PS assumes that the surface is strictly Lambertian. The Lambertian surface model states that light is observed at the same brightness in all directions regardless of the observation direction. Even though most surfaces are not strictly Lambertian, with a well-designed imaging system, non-Lambertian characteristics such as specularities can be minimized or excluded. The bidirectional reflectance distribution function (BDRF) is a mathematical model for observed brightness in a certain direction according to the placement of the light source and the surface normal. In a Lambertian surface, BDRF is simply a constant. Using BDRF, the observed image intensity $I$ can be formulated as in Equation 1.

$I=\rho_{d} N \bullet S$

where $\rho_{d}$ is the reflectance coefficient of the surface, also called as the albedo, $N$ is the surface normal vector and $S$ is the vector from the source to the surface. For multiple images Equation 1 can be rewritten as in Equation 2.

$\left[\begin{array}{c}I_{1} \\ \vdots \\ I_{n}\end{array}\right]=\left[\begin{array}{c}S_{1} \\ \vdots \\ S_{n}\end{array}\right] \rho N$

The least squares solution of Equation 2 is shown in Equation 3.

$G=\rho N$

$S^{T} I=S^{T} S G$

$G=\left(S^{T} S\right)^{-1}\left(S^{T} I\right)$ 
Because $N$ is a unit vector, albedo $\rho$ is calculated using 2-norm as given in Equation 4.

$\rho=\|G\|$

Finally, a normal vector can be obtained as in Equation 5.

$N=\frac{1}{\rho} G$

PS provides a powerful solution to problems that occur in traditional techniques that use only a single image. Advantages of using several images of the same surface under different lighting directions are as follows:

- No assumptions about the smoothness of the surface are required.

- Only additional light sources are needed.

- PS calculates not only the surface normal but also the albedo of the surface.

As a result of these advantages, PS is able to determine whether a darkening in the image of the object's surface is due to the shape of the object or to its albedo. To demonstrate, a metallic part with scratches that has also been drawn on with a marker is shown in Figure $4(\mathrm{a})$, and a processed image resulting from PS is shown in Figure 4(b).

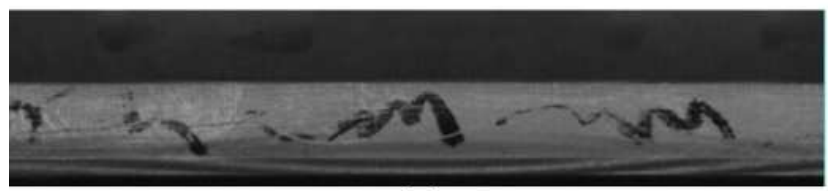

(a)

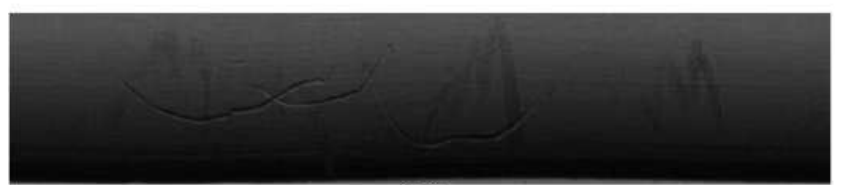

(b)

Fig. 4 (a)Image of the piece drawn on with a pen and knife (b)Gradient of the image which is the result of PS

\subsubsection{Deep Learning and $C N N$}

With the expansion of technology, the resultant increase in data has made deep learning one of the most important issues in recent years. Deep learning, which gives very successful results in many areas such as image classification, video analysis, voice recognition, and natural language processing, has an important place in the development of artificial intelligence today. The most important aspect of deep learning, which differs from classical machine learning approaches, is that it requires a large amount of data and highly computational hardware to process it. Due to the great improvements in the computing power of graphics cards, the processing of large amounts of data, especially in the field of image processing, yields successful results in a short time with deep learning algorithms. In contrast to traditional learning methods, deep learning is not based on any mathematical model that has already been created for feature extraction. Instead, attribute extraction is learned in deep learning layers. In this way, rather than a human deciding which type of attribute is appropriate for the data, the network to be used in deep learning and the operations to be applied in each layer gain importance.

Deep learning is becoming more popular with the ImageNet competition. Examples of successful CNN models are Le-Net, Alex-Net, ZF-Net, Google Net, and Microsoft-Net [25]. CNN is one of the deep learning algorithms that is widely used in image classification studies, and it gives very successful results. The convolution process reveals the specific features in an image. In the deep learning convolutional neural network, which has a multi-layered structure, a separate process is executed in each layer that then pushes the data to the next layer. Each layer performs its own function. Layers in the CNN architecture and their operations are explained in Section 2.1.3.

\subsubsection{Architecture of model}

CNN used in image processing consists mainly of convolutional, pooling, and fully connected layers. After receiving the input data in $\mathrm{CNN}$, feature exraction and training process are carried out. Finally, the network outputs a result to compare with the correct result. The difference between the generated result and the desired result is highlighted as an error. A backpropagation algorithm is used to update the weights for each layer sequentially. By updating the weights with each iteration, the error is reduced. The input data on the CNN can be any signal, such as images, audio, or video. Though focused mainly on image classification initially, CNN has been widely used in other fields in recent years.

Input Layer : In this layer, which is known as the first layer of $\mathrm{CNN}$, data is provided to the network for training.

Convolution Layer: This layer is based on the process of moving a particular filter over the entire image. Filters are therefore an integral component of the layered architecture. This layer allows us to look at each pixel of the image with the size and number of filters specified, so that hundreds of different patterns 


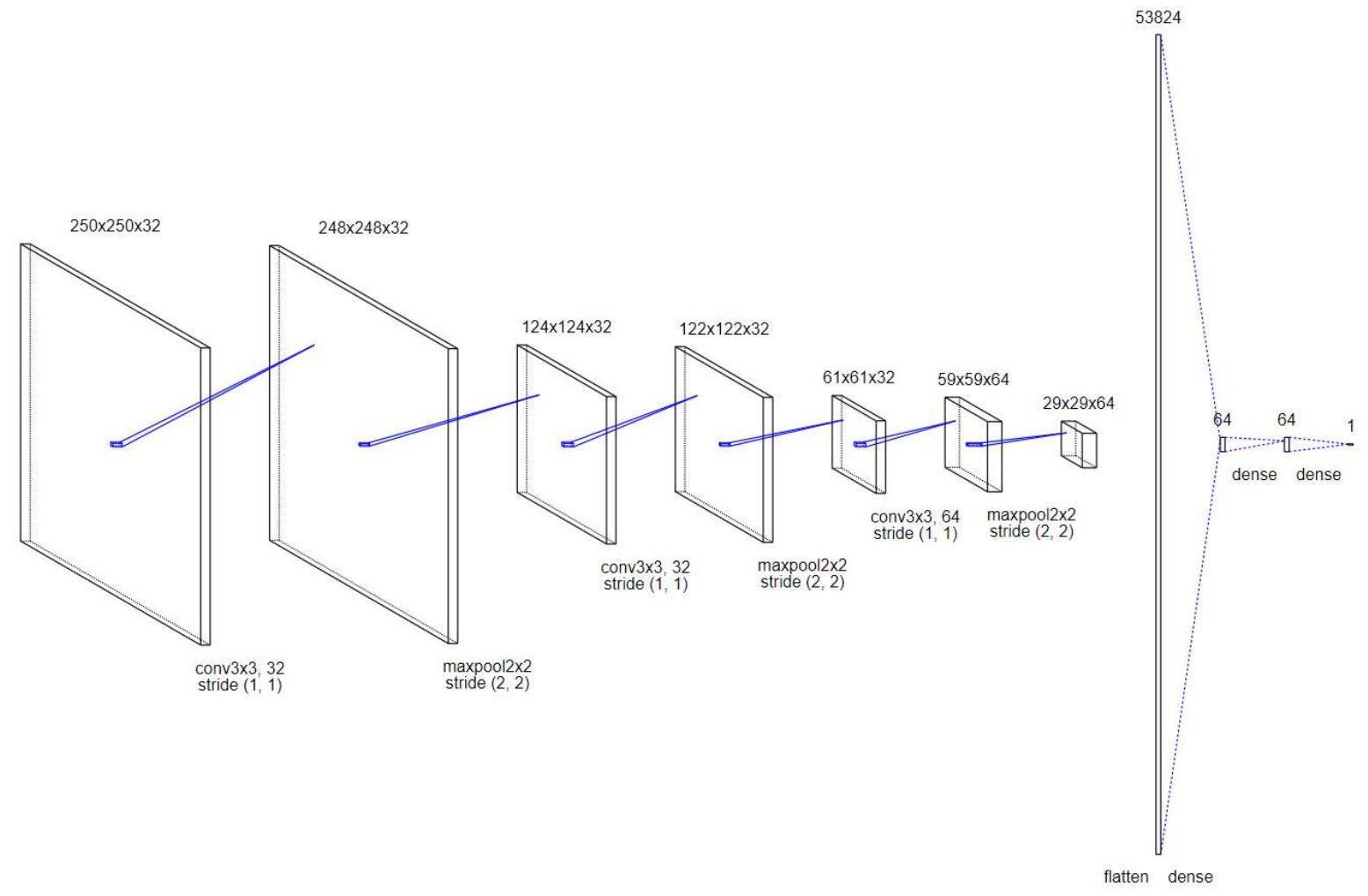

Fig. 5 Architecture of model

are found in the image. Figure S3 of the Supplementary file shows the convolutional layer.

Pooling Layer: The main purpose of this layer is to reduce the input size for the subsequent convolution layer to be created without affecting the depth dimension. Like the process in the convolution layer, certain filters are defined in the pooling layer and these filters are placed over the image according to a certain step value and process by taking the maximum values of the pixels in the image (maximum pooling) or the average of the values (average pooling). In this paper we used max pooling to defect detection of metalic parts since it introduces small invariance to translation and distortion, leads to faster convergence and better generalization [22]. Pooling is performed for all of the images by the number of filters formed by the convolution layer. Figure S4 of the Supplementary file shows the pooling layer.

Dropout Layer: When training with data in CNN, the network sometimes memorizes. This layer, which is used to prevent overfitting, is based on removing some nodes from the network.

Fully Connected Layer: This layer is the layer that allows us to find out which class the image belongs to.
The architecture of our network, which contains 11 layers, is summarized in Figure 5. A grayscale image is resized to contain $250 \times 250$ elements and then given to the first convolutional layer with 32 filter kernels of size $3 \times 3$. Then 32 feature maps of size $248 \times 248$ are produced. Next, the output of the first convolutional layer is taken as input by the first max pooling layer, whose kernel size is $2 \times 2$ with a stride of 2 . Then, 32 feature maps of size $124 \times 124$ comprise the output set. The second convolutional layer follows this with filter kernels of size $3 \times 3$. This results in 32 feature maps of size $122 \times 122$. Thereafter, the second convolutional layer is followed by the second max pooling layer, whose core size is $2 \times 2$ with a stride of 2 . An output set of 32 feature maps of size $61 \times 61$ is produced. Then, the third convolutional layer, which contains filter kernels of size $3 \times 3$ with a stride of 1 takes as input the output of the second max pooling layer. The third max pooling layer, whose kernel size is $2 \mathrm{x} 2$ with a stride of 2 , follows the third convolutional layer. After that, 64 feature maps of size $29 \times 29$ are created and linked into a $1 D$ feature vector of size $53824 \times 1$. This feature vector is subsequently fed into a classification layer, resulting in a 1 D output vector of size $64 \times 1$. 
Table 1 Metal defect detection accuracy for the proposed system compared with findings from other studies

\begin{tabular}{llc}
\hline Authors & Classifier & Overall Accuracy (\%) \\
\hline Ghorai et al. [18] & Support Vector Machines & 96,2 \\
Zheng et al. [19] & Genetic Algorithms & 88,5 \\
Malekian et al. [20] & Artificial Neural Networks & 96,5 \\
Proposed method & CNN & $\mathbf{9 8 , 3}$ \\
\hline
\end{tabular}

\section{Experiments}

\subsection{Training Details and Results}

The system was developed using the open source Keras Library in combination with NVIDIA's cuDNN library. Training was conducted on a conventional laptop with an NVIDIA GTX 850 M GPU card with 4GB memory.

The metal parts used in the study were separated as defective and non-defective based on visual inspection, and a total of 1516 images were obtained using the image acquisition system described in Chapter 2.1.

These images were separated into training and testing sets and prepared for entry into the CNN method. Of these images, $1212(80 \%)$ were used in training sets and 304 (20\%) were reserved for testing. Our data set was randomly distributed using a shuffling method. As a result of repeating this process five times, the overall accuracy values of training and testing were $98.34 \%$ and $98.00 \%$ respectively. The classification time of our system is five seconds.

Figure 6 shows graphs of accuracy values and loss values for the training and testing sets. As shown, the accuracy value fluctuates within a narrow range between $98 \%$ and $99 \%$. Training accuracy is over $98 \%$ and testing accuracy is more than $97.5 \%$. Loss value is close to 0 in both the training and the testing set.

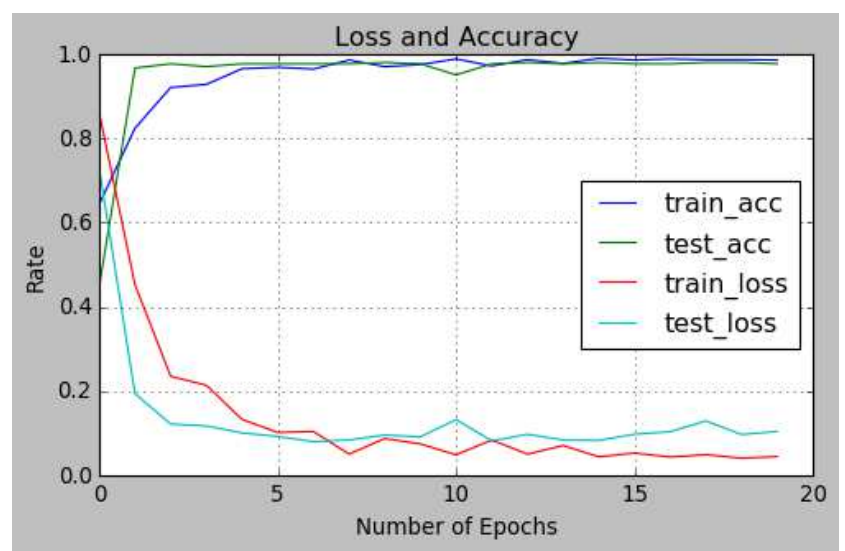

Fig. 6 Training and Testing Accuracy - Loss Graphics

Of the 304 data items allocated for the testing set, 135 of 136 Nok (defective) items were classified successfully, while only five of $168 \mathrm{Ok}$ (non-defective) data were misclassified. The confusion matrix of our system, which gives very successful results, is shown in Figure 7.

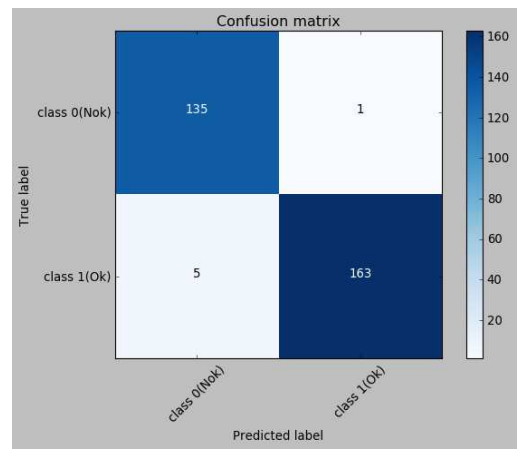

Fig. 7 Confusion Matrix of Our System

The confusion matrix table and accuracy-loss graphs showing the success rates of the training are described above, with optimizer rmsprop and loss function cross entropy selected for 1212 training items and 304 test items. The most successful results occurred at 20 epochs.

As a result of the convolution process, the specified matrices are placed over the entire image and the features in the image are clarified. Figure S5 of the Supplementary file shows images of the metal created using different filters with a convolution layer.

The performance of the proposed system was compared to metallic surface defect detection studies in the literature. Table 1 shows the comparison results and it is seen that our study yielded successful results.

\section{Conclusions}

Currently, one of the most common sectors for automation and mass production is the automobile sector. Today's car industry needs high precision parts. Therefore, it would be desirable for the level of fault in auto parts to be reduced to zero. Generally, in mass production, quality control should be managed with computers, especially through the use of computer vision. However, quality control for metal parts is a difficult problem to resolve with this technology. In this study, computer 
vision for quality control of metallic car parts was performed using deep learning within quality control software.

In this study we used PS to derive surface normals from images of a surface created under different illumination directions, and the shape of the surface was calculated from these normals. PS was used to render a single image of each metallic surface strip based on images taken from four different angles. Then these images were provided to CNN as input data. The study used 1516 images, of which 1212 were employed in training and 314 in testing. The system was found to have a success rate close to $98.5 \%$ and was developed to be used in the production of a series of parts where quality control is of the essence, particularly in the automotive industry. Our defect detection system can be expanded and used to locate defective areas of other kinds in subsequent studies.

\section{Acknowledgments}

This work was supported by the TUBITAK (The Scientific and Technological Research Council of Turkey) 1505 and was carried out in cooperation with Cengiz Machine Company. The authors sincerely thank the project team members of TUBITAK 1505 for their encouraging and constructive feedback.

\section{Availability of data and materials}

The study was carried out with our special data set belonging to our joint project with TUBITAK.

\section{Competing Interest}

The authors declare that they have no competing interests.

\section{Authors' contributions}

FC , SK and C.O were in charge of the whole trial and wrote the manuscript; MAO assisted with the use of software. T.T, S.H and C.A manufactured the prototype and conducted the test. All authors read and approved the final manuscript.

\section{Funding}

Supported by the TUBITAK (The Scientific and Technological Research Council of Turkey) 1505

\section{References}

1. S Pan, T Y Hung, and L T Chia. Using material classification methods for steel surface defect inspection. IEEE Int. Symp. Ind. Electron, pages 40-45, 2016.

2. Q Sun, J Cai, and Z Sun. Detection of Surface Defects on Steel Strips Based on Singular Value
Decomposition of Digital Image. Math. Probl. Eng, 2016:1-12, 2016.

3. R Gong, M Chu, A Wang, and Y Yang. A Fast Detection Method for Region of Defect on Strip Steel Surface. ISIJ Int, 55(1):207-212, 2015.

4. E A Kholief, S H Darwish, and M N Fors, (2017).

5. R Alkapov, A Konyshev, N Vetoshkin, N Valkevich, and P Kostenetskiy. Automatic Visible Defect Detection and Classification System Prototype Development for Iron-and-Steel Works. Proc. - 2018 Glob. Smart Ind. Conf. GloSIC, 2018.

6. X Sun, J Gu, S Tang, and J Li. Research Progress of Visual Inspection Technology of Steel ProductsA Review. Appl. Sci, 8(11):2195-2195, 2018.

7. M Arun, R Prathipa, and P S G Krishna. Automatic Defect Detection of Steel Products Using Supervised Classifier. Int. J. Innov. Res. Comput. Commun. Eng, pages 3630-3635, 2014.

8. E Pishyar and M Emadi. Investigation of Different Algorithms for Surface Defects of Steel Sheet for Quality. Int. J. Comput. Appl, 149(6):33-37, 2016.

9. O Essid, H Laga, and C Samir. Automatic detection and classification of manufacturing defects in metal boxes using deep neural networks. PLoS One, 13(11), 2018.

10. H S Bar. Real-Time Defects Detection Algorithm for. Eng. Technol, 1(1):66-70, 2007.

11. M Muehlemann. Standardizing Defect Detection for the Surface Inspection of Large Web Steel, 2000. Technol.

12. R J Woodham. Photometric method for determining surface orientation from multiple images. Opt. Eng, 19(1):191139-191139, 1980.

13. Lei Wang, Ke $\mathrm{Xu}$, and Peng Zhou. Online detection technique of 3D defects for steel strips based on photometric stereo. Eighth International Conference on Measuring Technology and Mechatronics Automation (ICMTMA), 2016.

14. Gregor Podrekar. Model based visual inspection of pharmaceutical tablets with photometric stereo. Fifteenth IAPR International Conference on $M a-$ chine Vision Applications (MVA), 2017.

15. Mingjun Ren. Model-driven photometric stereo for in-process inspection of non-diffuse curved surfaces. CIRP Annals, 2019.

16. M Ren, X Wang, G Xiao, M Chen, and L Fu. Fast Defect Inspection Based on Data-Driven Photometric Stereo. IEEE Transactions on Instrumentation and Measurement, 68(4):1148-1156, 2018.

17. Jong Lee, Hyun Min Hyuk, Min Young Oh, and Kim. Deep learning based 3D defect detection system using photometric stereo illumination. 2019 International Conference on Artificial Intelligence in 
Information and Communication (ICAIIC), 2019.

18. S Ghorai, A Mukherjee, M Gangadaran, and P K Dutta. Automatic defect detection on hot-rolled flat steel products. IEEE Trans. Instrum. Meas, $62(3): 1-9,2000$.

19. H Zheng, L X Kong, and S Nahavandi. Automatic inspection of metallic surface defects using genetic algorithms. J. Mater. Process. Technol, pages 427433, 2002.

20. Vahid Malekian, Rassoul Amirfattahi, Mahdie Rezaeian, Alireza Aghaei, and Pooian Rahimi. Automatic detection and localization of surface cracks in continuously cast hot steel slabs using digital image analysis techniques. International Journal of Iron E6 Steel Society of Iran, pages 30-40, 2012.

21. S Zhou, Y Chen, D Zhang, J Xie, and Y Zhou. Classification of surface defects on steel sheet using convolutional neural networks. Mater. Tehnol, 51(1):123-131, 2017.

22. J Masci, U Meier, D Ciresan, J Schmidhuber, and $G$ Fricout. Steel defect classification with MaxPooling Convolutional Neural Networks. Proc. Int. Jt. Conf. Neural Networks, 2012.

23. R Ye, C S Pan, M Chang, and Q Yu. Intelligent defect classification system based on deep learning. Adv. Mech. Eng, 10(3):1-7, 2018.

24. Laila Ma'rifatul Azizah, Sitti Fadillah Umayah, Slamet Riyadi, Cahya Damarjati, and Nafi Ananda Utama. Deep learning implementation using convolutional neural network in mangosteen surface defect detection. pages 242-246, 2017.

25. Dina Abdelhafiz, Clifford Yang, Reda Ammar, and Sheida Nabavi. Deep convolutional neural networks for mammography: advances, challenges and applications. BMC bioinformatics, 20:281, 2019. 


\section{Figures}

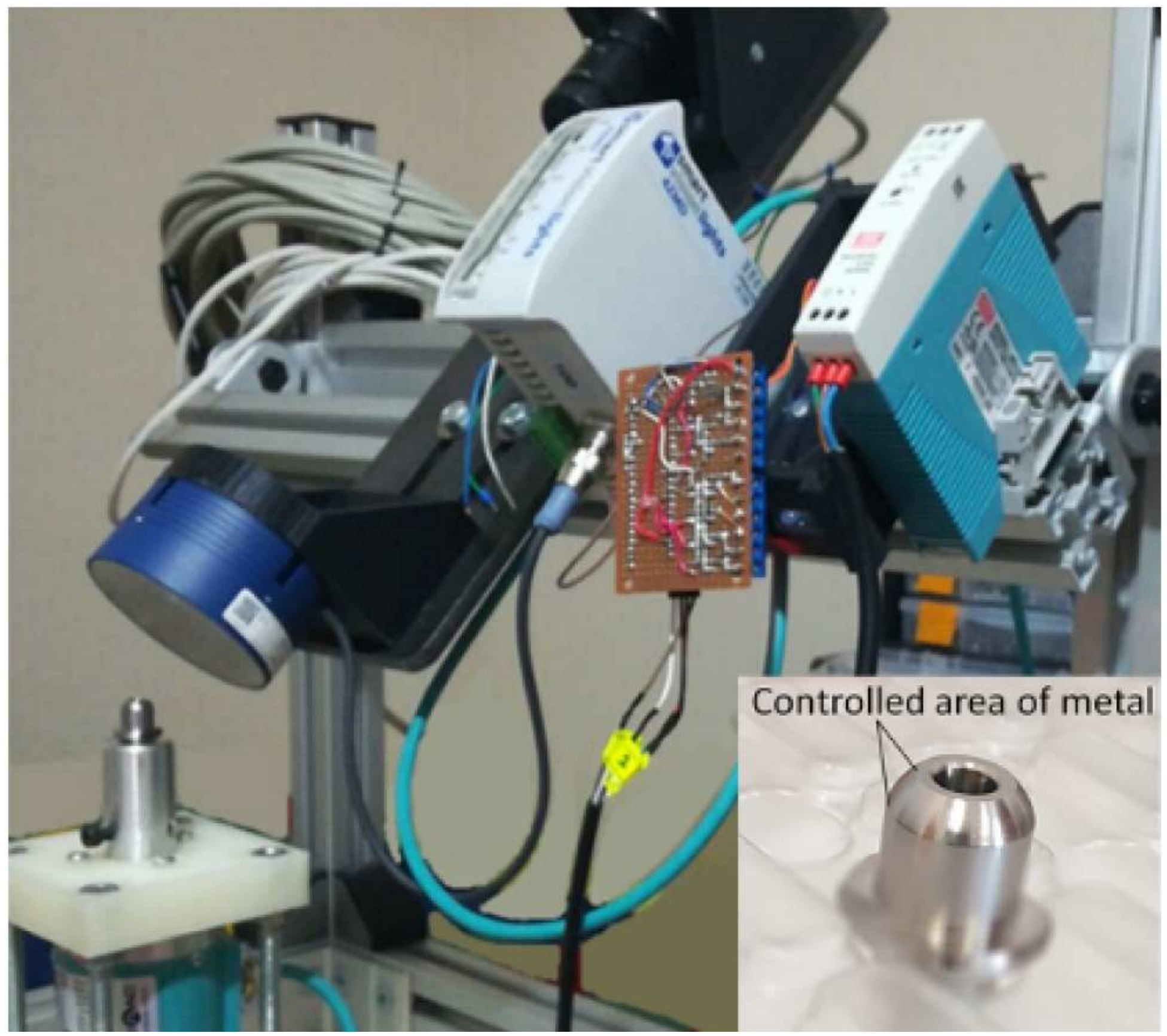

Figure 1

Image acquisition system and controlled area of metal 


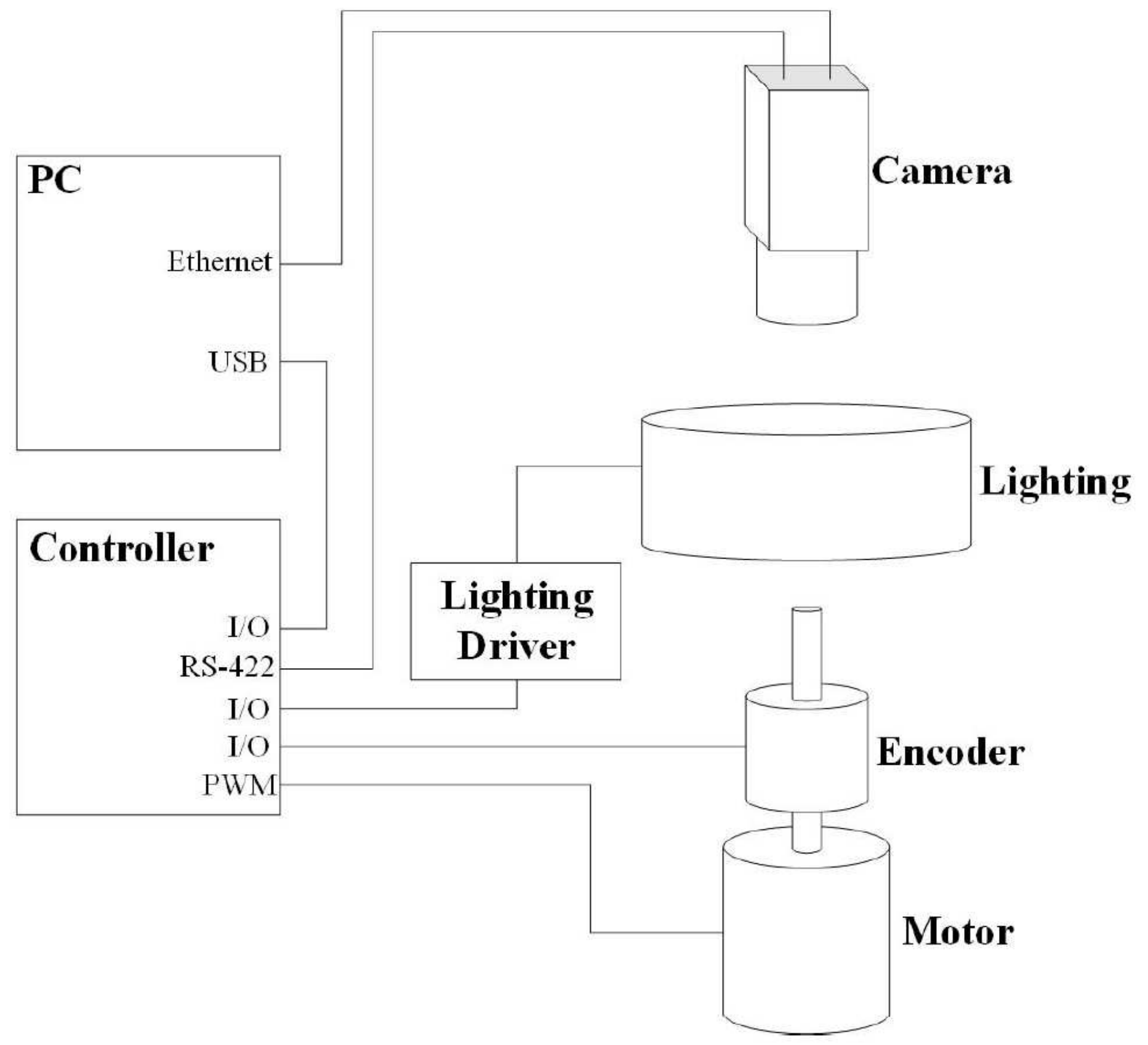

Figure 2

Block diagram of the system 

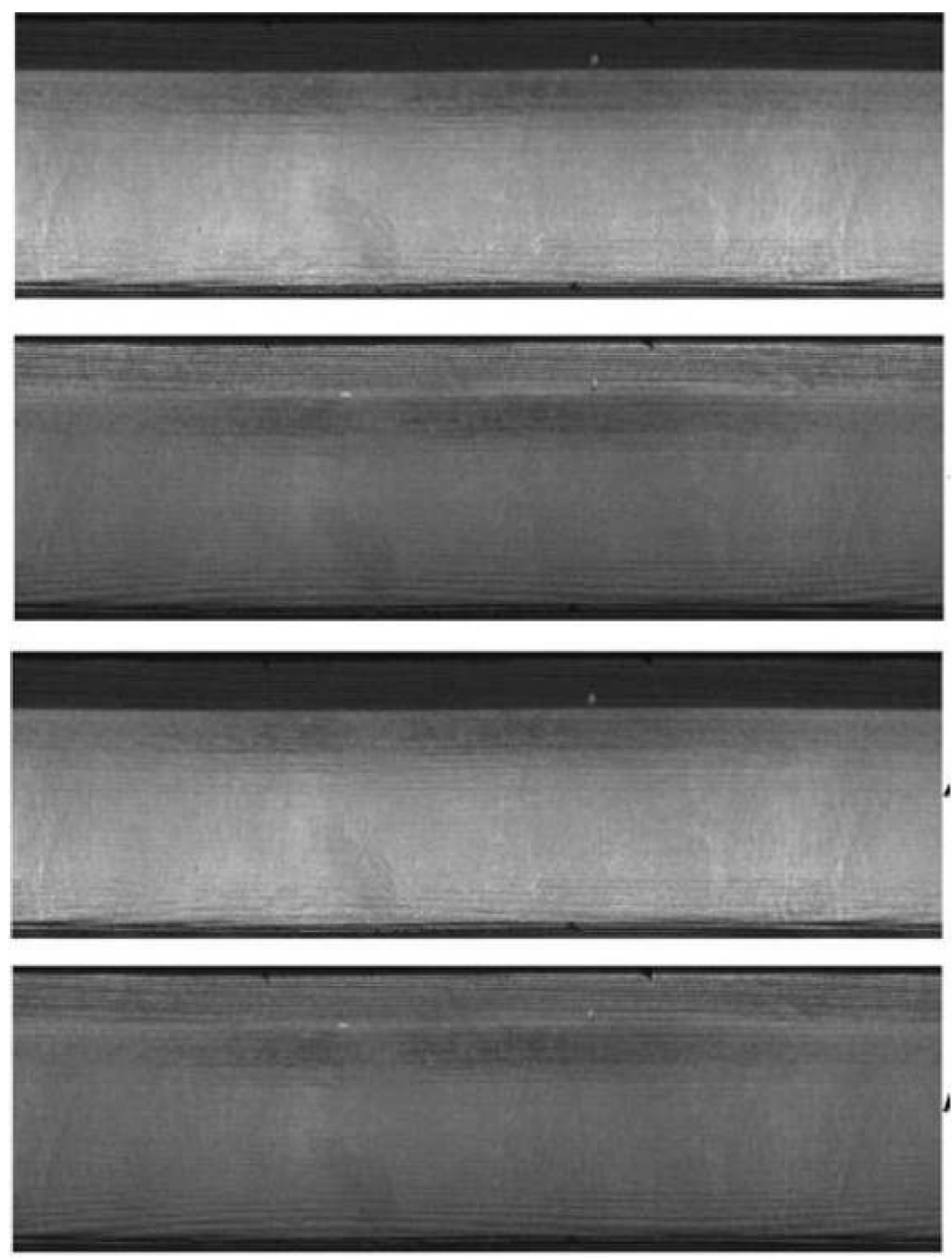

Figure 3

Four images produced after pre-process phase 


\section{(a)}

\section{(b)}

\section{Figure 4}

(a)Image of the piece drawn on with a pen and knife (b)Gradient of the image which is the result of PS 


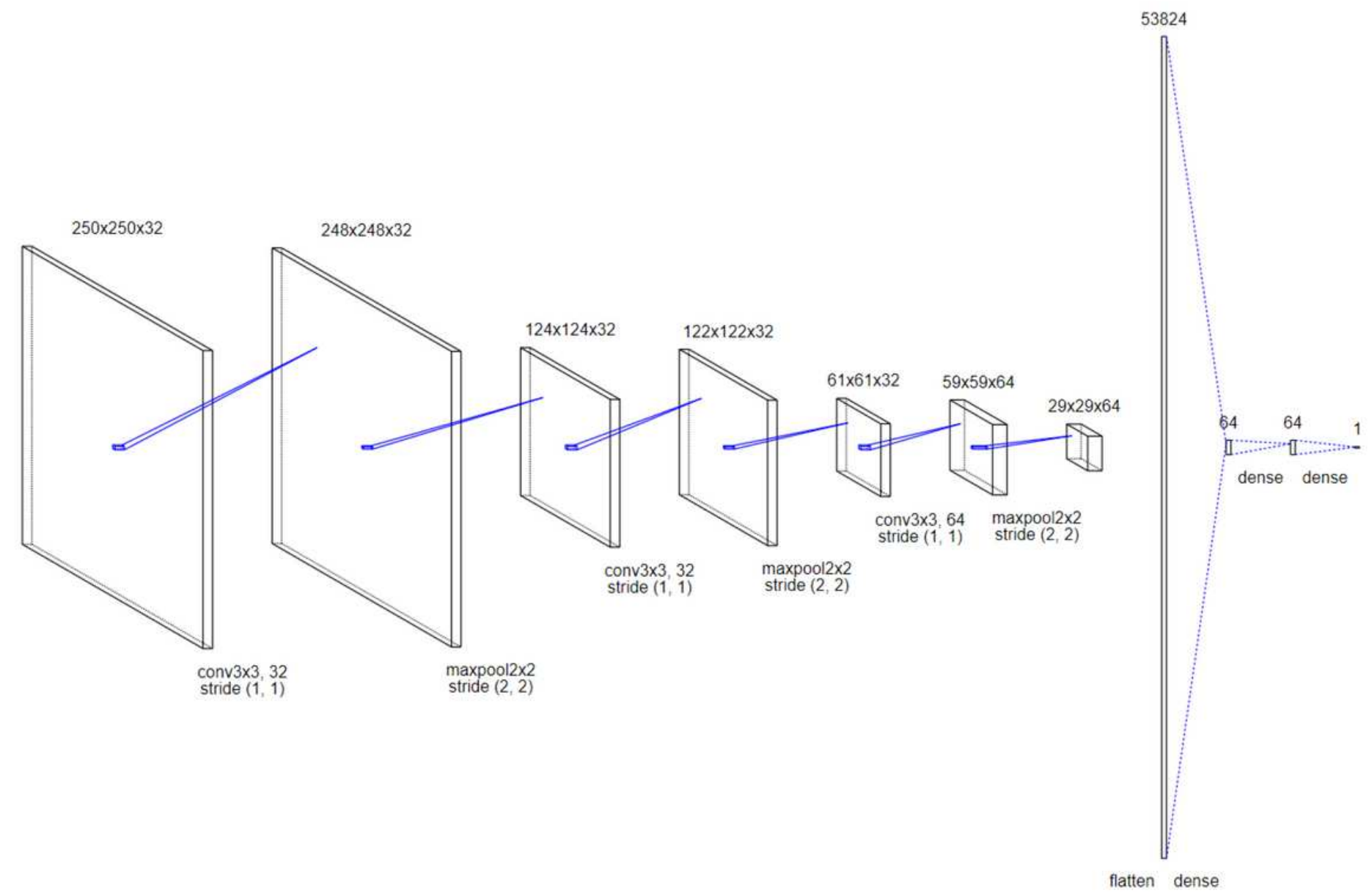

Figure 5

Architecture of model 


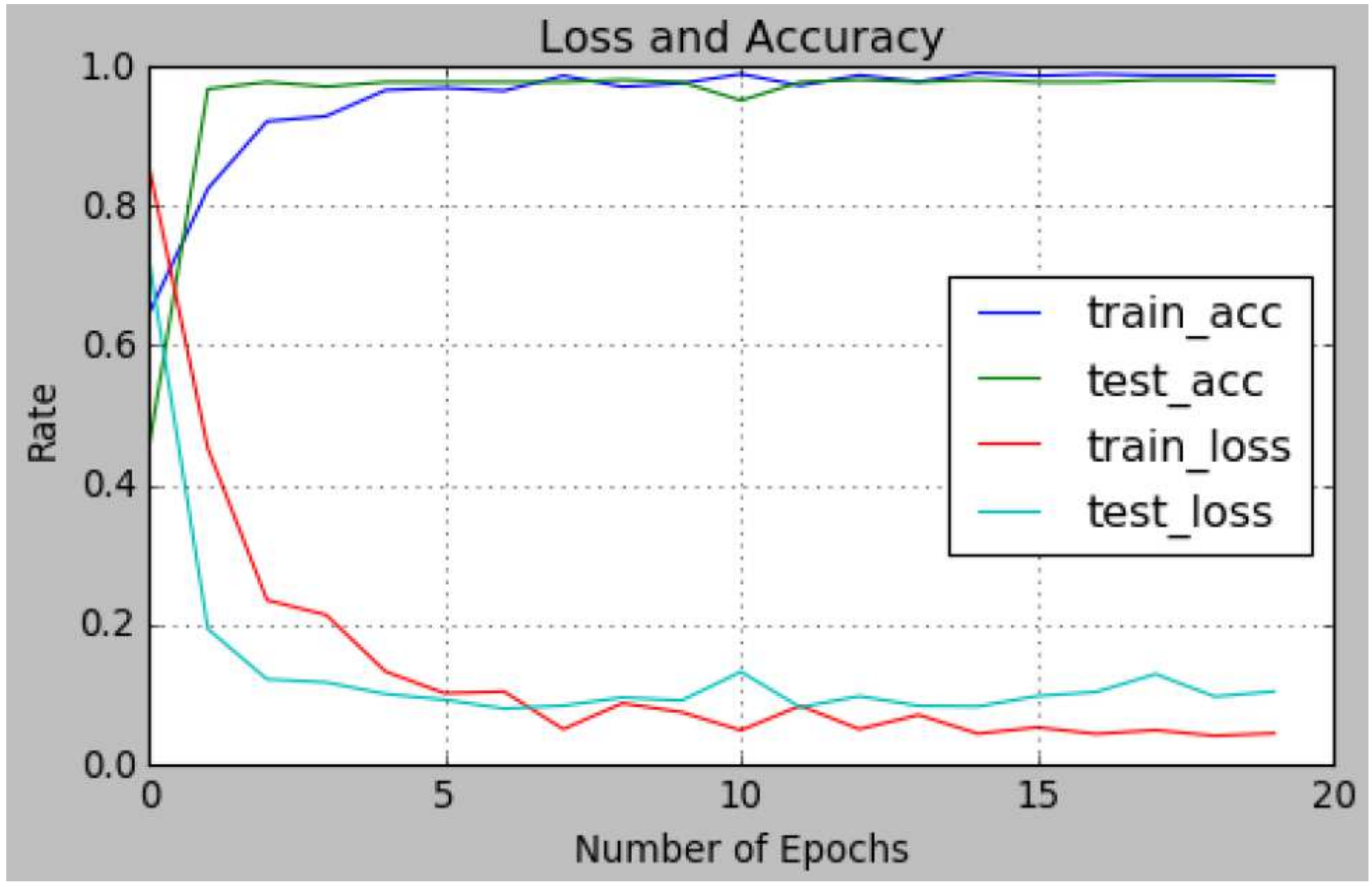

Figure 6

Training and Testing Accuracy - Loss Graphics 


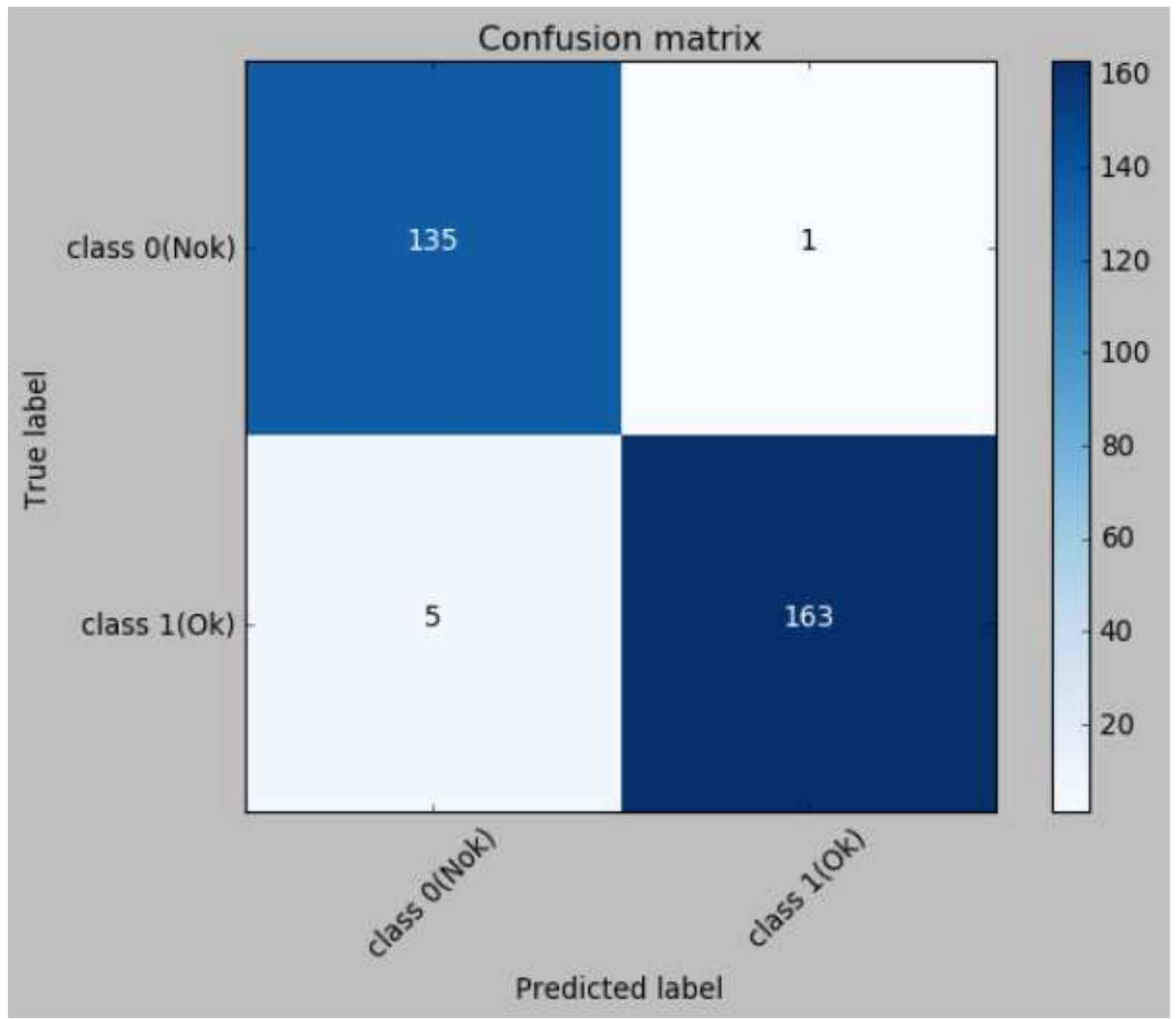

Figure 7

Confusion Matrix of Our System

\section{Supplementary Files}

This is a list of supplementary files associated with this preprint. Click to download.

- SupplementaryMaterial.pdf 